\title{
Red mulberry fruit aqueous extract and silk proteins accelerate acute ethanol metabolism and promote the anti-oxidant enzyme systems in rats
}

\author{
HYE JEONG YANG ${ }^{1}$, MIN JUNG KIM ${ }^{1}$, EUN SEON KANG ${ }^{2}$, DA SOL KIM ${ }^{2}$ and SUNMIN PARK ${ }^{2}$ \\ ${ }^{1}$ Food Functional Research Division, Korean Food Research Institutes, Wanju, Jeollabuk 55365; ${ }^{2}$ Department of \\ Food and Nutrition, Obesity/Diabetes Center, Hoseo University, Asan, South Chungcheong 336-795, Republic of Korea
}

Received December 17, 2017; Accepted April 26, 2018

DOI: $10.3892 / \mathrm{mmr} .2018 .9017$

\begin{abstract}
Red mulberry (Morus alba) fruit is rich in anthocyanins, and mulberry leaves are used by silk worms to make silk protein. We determined that the water and ethanol extract of mulberry fruit and silk amino acids accelerated ethanol degradation and suppressed temporal cognitive dysfunction in acute alcohol administered rats. The mechanism was explored in rats with acute oral administration of silk protein and mulberry fruit extracts. Rats were given $0.3 \mathrm{~g}$ of dextrin (control) and water extract (WMB) and ethanol extract of mulberry (EMB), silk protein hydrolysates (SKA), and a commercial product (positive-control) based on body weight. After $30 \mathrm{~min}$, rats were administered $3 \mathrm{~g}$ ethanol/kg body weight and serum ethanol and acetaldehyde levels were measured. After $3 \mathrm{~h}$ movements were measured with a video tracking system and at $5 \mathrm{~h}$ cognitive function was measured by $\mathrm{Y}$ maze test. WMB contain much higher rutin, luteolin and quercetins than EMB. In SKA rats, serum alcohol concentrations slowly increased until $60 \mathrm{~min}$, but were markedly elevated until $120 \mathrm{~min}$. However, WMB rats exhibited rapidly increased serum alcohol levels until $60 \mathrm{~min}$
\end{abstract}

Correspondence to: Dr Sunmin Park, Department of Food and Nutrition, Obesity/Diabetes Center, Hoseo University, 165 Sechul-Ri, BaeBang-Yup, Asan, South Chungcheong 336-795, Republic of Korea

E-mail:smpark@hoseo.edu

Abbreviations: NAD+, nicotinamide adenine dinucleotide; $\mathrm{ADH}$, alcohol dehydrogenase; ALDH, acetaldehyde dehydrogenase; SIRT, sirtuin; CYP2E1, cytochrome P450 2E1; control, the groups administered dextrin; WMB, the group administered water extract of mulberry; EMB, administered ethanol extract of mulberry; SKA, the group administered silk protein hydrolysates; ALT, alanine aminotransferase; AST, aspartate aminotransferase; $\gamma$-GPT, $\gamma$-glutamyl transpeptidase; TBARS, thiobarbituric acid reactive substance; TG, triglyceride; TNF- $\alpha$, tumor necrosis factor- $\alpha$; GSH, glutathione; $\mathrm{Cq}$, cycle of quantification

Key words: ethanol, acetaldehyde, alcohol dehydrogenase, acetaldehyde dehydrogenase, lipid peroxides and showed the lowest peak of serum alcohol levels, indicating the highest degradation of alcohol. The patterns of serum acetaldehyde levels were similar to those of serum ethanol levels but WMB was more effective for reducing serum acetaldehyde levels than serum ethanol levels. WMB was most effective for increasing mRNA expression of alcohol dehydrogenase and acetaldehyde dehydrogenase. WMB and SKA decreased lipid peroxides by increasing activities of SOD and GSH-Px in the liver and they also reduced pro-inflammatory cytokines such as tumor necrosis factor- $\alpha$ and interleukin-6. WMB and SKA exerted better anti-oxidant effects than the positive-control. WMB containing higher flavonoids reduced pro-inflammatory cytokines better than SKA. In conclusions, both WMB and SKA might reduce acute alcohol-induced hangover and liver and brain damage by lowering serum alcohol and acetaldehyde levels.

\section{Introduction}

Mild to moderate alcohol consumption has some beneficial physiological and psychological effects. However, consuming large amounts of alcohol and can cause acute alcohol-induced stress referred to as a hangover, and chronic alcohol consumption can lead to liver and multi-organ damage. Ethanol is metabolized into acetaldehyde in the intestinal tract and liver, mainly by alcohol dehydrogenase (ADH), and is absorbed in the stomach and intestinal tract with the remaining ethanol oxidized to acetaldehyde in the liver (1). Acetate is used as energy source and may be converted fat and stored in the body. Minimal amounts of alcohol are removed as alcohol itself through sweat, urine, and breath.

During alcohol degradation, NADH, the reduced form of nicotinamide adenine dinucleotide $\left(\mathrm{NAD}^{+}\right)$, is produced by $\mathrm{ADH}$ and acetaldehyde dehydrogenase (ALDH), which suppresses lipid metabolism in the liver and intracellular $\mathrm{NAD}^{+}$levels are decreased (2). Acetaldehyde is very toxic to the hepatic cells and it induces hangover. In addition, unmetabolized alcohol by ADH is degraded by cytochrome P450 in a process that forms oxygen radicals such as superoxides which in turn generate lipid peroxides $(3,4)$. Increased oxidative stress damages hepatic cells by inactivating enzymes that metabolize fats, facilitate lipoprotein production and disrupt 
cell membranes. In addition, $\mathrm{NAD}^{+}$is a coenzyme for sirtuin (SIRT), an NAD ${ }^{+}$-dependent deacetylase, and the decrease in NAD ${ }^{+}$levels suppresses SIRT activity (2). The decreased expression and activity of SIRT1 and SIRT3 causes the induction of enzymes related to lipogenic pathways, inflammatory response, and oxidative stress and decreases the enzymes related to fatty acid oxidation and fat mobilization (2). As a result, endogenous and exogenous fat cannot be incorporated into very low density lipoprotein in the liver and fat is trapped in the liver. Chronic alcohol consumption leads to alcohol-induced hepatosteatosis and it also induces pancreatitis, neuropathy, liver cirrhosis, myocardial infarction and cancer (5).

Acute heavy drinking causes hangover symptoms such as headache, vomiting, dizziness, dehydration and muscle pain, mostly due to the acetaldehyde. Acetaldehyde easily crosses the blood brain barrier and it is changed into harmful compounds that reduce blood volume by increasing urination and increase oxidative stress (6). Ethanol toxicity can be decreased by effectively decreasing serum ethanol and acetaldehyde concentrations after alcohol consumption. In addition, lipid peroxides and inflammatory cytokine concentrations need to be lowered to relieve ethanol toxicity $(7,8)$. Therefore, alcohol needs to be quickly degraded into acetate and the generation of free radicals and pro-inflammatory cytokines needs to be suppressed during alcohol degradation.

People have used coffee, tea, ion drinks, vitamin B6, and pain killers and several hangover drinks to relieve the symptoms of ethanol toxicity. Several herbs have been scientifically evaluated for efficacy in accelerating alcohol degradation and decreasing oxidative stress and inflammation (9-12). Pear juice, red ginseng, asparagine in bean sprouts, and Hovenia dulcis Thunb have been studied for improving alcohol metabolism and reducing hangover (9-12). Leaves, bark and fruits of Morus alba L. have been reported to have hypoglycermic and hypocholesterolemic activities and silk protein that is made of cocoon also have anti-diabetic effects. Mulberry fruits and silk protein are the family of Morus alba. Mulberry contains anthocyanins that are well-known to have anti-oxidant and anti-inflammatory activities. Jiang et al (13) reported that purple potato containing anthocyanins increase cytochrome P450 2E1 (CYP2E1) activity, and thereby strengthens antioxidant defenses in alcohol-induced liver damage. Anthocyanins from black rice also protect against alcohol-induced liver damage in rats by improving lipid metabolism and the anti-oxidant system (14). In addition, silk protein hydrolysates (SKA) have been shown to protect against liver damage induced by alcohol and carbon tetrachloride. $(15,16)$ Silk protein improved lipid metabolism and decreased gluconeogenesis while also potentiating the anti-oxidant system $(15,17)$. Thus, mulberry fruits and silk protein, a cocoon lysis product, may reduce hangover and protect against liver damage by alcohol. However, no studies have conducted to investigate whether either mulberry or silk protein modulates alcohol degradation and hangover in a short-term study.

Here, we hypothesized that the water and ethanol extract of mulberry and silk amino acids, might accelerate ethanol degradation and suppress temporal cognitive dysfunction by potentiating the anti-oxidant system in acute alcohol administered rats. The hypothesis was tested in acute alcohol-administered rats supplemented with silk protein and mulberry fruit extracts.

\section{Materials and methods}

Water extract of mulberry and silk protein. Mulberry fruits (Buan, Chonbuk, Korea) were homogenized and the homogenates were extracted in 3-fold volume of at $60^{\circ} \mathrm{C}$ for $2 \mathrm{~h}$. The extract was filtered and concentrated up to $50 \%$ using a low-pressure rotary evaporator. The supernatant was separated by centrifugation at $8,000 \times \mathrm{g}$ for $30 \mathrm{~min}$. and freeze-dried to make a powder.

Dried silkworm (Bombys mori) cocoon hydrosylates were obtained from Worldway Co., Ltd. (Sejong, Korea) and stored for the further study. The silkworm cocoons were prepared by washing with 13-15 volume of water and hydrolyzing with $2 \mathrm{~N} \mathrm{HCl}$ at $100-110^{\circ} \mathrm{C}$ for $12 \mathrm{~h}$. The hydrolysates were filtered and salt contents in the hydrolysates were lowered to less than $0.3 \%$ at $\mathrm{pH}$ 5.5-7.5. The acid hydrolysates were sterilized and concentrated to 20-25 Brix in a low-pressure evaporator and then dried by a spray dryer.

Animals and experimental design. All surgical and experimental procedures were performed according to the guidelines and with the approval of the Animal Care and Use Review Committee at Hoseo University, Korea (2013-05). Male Sprague Dawley rats aged of 7-8 weeks were purchased from Daehan Biolink (Eum Sung, Korea) and they were housed in stainless steel cages in a controlled environment: Temperature $\left(22 \pm 1^{\circ} \mathrm{C}\right)$, humidity $(55 \pm 4 \%)$, and a $12 \mathrm{~h}$-light/dark cycle. After a 1-week acclimation in the animal facility, the 50 rats were divided into the following 5 treatment groups: Dextrin (control), water extract of mulberry (WMB), ethanol extract of mulberry (EMB), SKA, and a commercial hangover product: Condition, made from extracts of Hovenia dulcis Thunb fruits (CJ, Seoul, Korea; positive-control). Assigned extracts were orally administered to each rat by dissolving $0.3 \mathrm{~g}$ dried extracts into $1 \mathrm{ml}$ water, except for the positive-control. WMB, EMB and SKA was equivalent to about $2.5 \mathrm{~g}$ a time as a human dosage. Since 'Condition' need to provide $12 \mathrm{ml} / \mathrm{kg}$ body weight, the product was concentrated in vaccum evaporator by 12 folds and then gave $1 \mathrm{ml}$ as a positive-control group.

For determining the effect of mulberry extracts and silk hydrolysates on alcohol degradation in the body, the assigned extracts were orally given to the rats in each group and after $30 \mathrm{~min}$ the rats were administered $3 \mathrm{~g}$ ethanol/ $\mathrm{kg}$ bw by oral gavage. The amount of ethanol to be provided was equivalent to 25-30 g ethanol for human. The rats were allowed no additional water or food and blood samples were taken from tail vein at $0.5,1,3$, and $5 \mathrm{~h}$. After the final blood collection, the rats were provided food and water ad libitum. At 3 days later, the rats were subjected to the same experiment of alcohol administration following extract administration. Blood samples collected during the alcohol experiment were allowed to sit for $20 \mathrm{~min}$ on ice to coagulate and were then centrifuged at 1,500 x g for $20 \mathrm{~min}$ and serum was separated. Serum ethanol and acetaldehyde concentrations were measured by colorimetric methods using ethanol (BioVision, Milpitas, CA, USA) and aldehyde (Abcam, Cambridge, MA, 
USA) quantification kits according to the manufacturer's instructions.

Movement and Y maze tests. Alcohol consumption affects the brain and elevated serum ethanol levels induce lethargy and reduced movement. Movement was monitored by a video tracking system (Ethovision system; Noldus, Wageningen, Netherlands) for $10 \mathrm{~min}$ at $3 \mathrm{~h}$ after ethanol administration and moving distance and velocity of movement were measured. In addition, ethanol affects spontaneous alternation performance which was assessed by using a Y maze test at $5 \mathrm{~h}$ after orally administering ethanol (18). As a rat remembers the previous location, the rat's pathway rotates through the Y maze. A rat was placed into one of the arm compartments (usually arm A for consistency) in the Y maze and for $10 \mathrm{~min}$, and was allowed to freely explore the $\mathrm{Y}$ maze. The arm entry was scored when the rat remained in an arm of the Y-maze. An alternation is defined as an entry into all three arms in consecutive order. The sequence of arm entries was recorded. The percentage alternations was calculated as the following formula: (Total alternation number/Total number of entries-2) x100. The Y maze arms was cleaned with $70 \%$ ethanol between each trial.

Biochemical assays. After collecting $5 \mathrm{~h}$ blood, rats were sacrificed under anesthesia with ketamine and xylazine (100 and $10 \mathrm{mg} / \mathrm{kg}$ bw, respectively) since serum ethanol levels had almost returned to the baseline and any damage by ethanol was completed. Blood was intraperitoneally taken from each rat and liver was dissected. Serum was separated after centrifugation of the blood. The serum levels of alanine aminotransferase (ALT), aspartate aminotransferase (AST) and $\gamma$-glutamyl transpeptidase $(\gamma$-GPT), markers for liver damage, were measured by colorimetric methods using kits obtained from Asan Pharmaceutical company (Seoul, Korea). Livers and brains were collected and stored at $-70^{\circ} \mathrm{C}$ for further study.

Antioxidant status. Lipid peroxide levels in the liver and brain were measured using a thiobarbituric acid reactive substance (TBARS) assay kit (Cayman Chemical, Ann Arbor, Michigan, USA). Triglyceride (TG) contents were also measured in the liver and brain using a TG kit (Asan Pharmaceutical, Seoul, Korea). Tumor necrosis factor- $\alpha$ (TNF- $\alpha$ ) levels in the DMEM media were measured using ELISA kits (R \& D Systems, Minneapolis, MN and Amersham Biosciences, Piscataway, NJ, USA respectively). The activities of anti-oxidant enzymes such as $\mathrm{Cu} / \mathrm{Zn}$ superoxide dismutase (SOD) and glutathione (GSH)-peroxidase were measured from the lysates of the liver tissues by using colorimetry kits (Cayman Chemical, Ann Arbor, Michigan, USA and Biovision, Milpitas, CA, USA), respectively. One unit of each enzyme activity was defined as $50 \%$ inhibition of each enzyme reaction and the enzyme activity was normalized by $\mathrm{mg}$ protein in the lysate.

Reverse transcriptase-quantitative polymerase chain reaction. The liver tissues from five randomly selected mice from each group were collected at the end of the experimental period. Total RNA was isolated from the skin tissues and cells with a monophasic solution of phenol and guanidine isothiocyanate (Trizol reagent, Invitrogen, Rockville, MD, USA). The cDNA was synthesized with a mixture of equal amounts of total RNA, superscript III reverse transcriptase and high fidelity Taq DNA polymerase for polymerase chain reaction (PCR). The expressions of the genes of interest were measured from the mixture of cDNA, primers of the genes of interest and sybergreen mix using a realtime PCR machine (BioRad Laboratories, Hercules, CA, USA). The primers for TNF- $\alpha, \mathrm{ADH}$, aldehyde dehydrogenase and $\beta$-actin were given in previous studies $(19,20)$. The gene expression levels in each sample were quantitated using the comparative cycle of threshold (Cq) method (21).

Statistical analysis. Statistical analysis was performed using SAS software and all results were expressed as mean \pm standard deviation. The variables related to the metabolic changes were compared among control, WMB, EMB, SKA, and positive-control by one-way analysis of variance (ANOVA) in cell-based and animal studies. Multiple comparisons among the groups were conducted by Tukey's test at $\mathrm{P}<0.05$.

\section{Results}

Bioactive components of various extracts of mulberry and $S K A$. WMB and EMB contained various polyphenols such as hydroxybenzoic acid, genestic acid, rutin, luteolin, cinamic acid and cyanidin-3-glycosides (Table I). They also had 4-aminobutanoic acid (GABA). WMB contained higher amounts of the bioactive compounds, especially flavonoids and anthocyanins than EMB (Table I). SKA contained most amino acids, but was especially rich in glycine and alanine (Table II).

Serum ethanol and acetaldehyde concentrations after ethanol administration. Since ethanol drinking is a repeated behaviour in real life and the repetition itself affects ethanol metabolism, the effects of mulberry extracts and silk protein were examined twice, at three days apart. Rats were orally administered ethanol after the assigned extracts were orally administered. Serum ethanol concentration increased until 120 min after ethanol administration, and then they began declining (Figs. 1 and 2). Serum acetaldehyde concentrations also increased until $120 \mathrm{~min}$ and they decreased after $120 \mathrm{~min}$ (Figs. 1B, 2B). Rats given WMB and EMB experienced much lower increases in serum alcohol concentrations after $30 \mathrm{~min}$ than the control group until $120 \mathrm{~min}$. Serum alcohol concentrations also decreased in WMB and EMB treated rats more rapidly than in the control group after 120 min (Figs. 1A, 2A). WMB lowered serum alcohol levels as much as the positive control group in the 1st trial (Fig. 1A) but WMB reduced serum alcohol levels more than the positive-control in the 2 nd trial (Fig. 2A). SKA markedly delayed the increase in serum alcohol levels especially for the first $60 \mathrm{~min}$ but the decrease of serum alcohol levels was not accelerated after $120 \mathrm{~min}$. This indicated that SKA might slow alcohol absorption and that alcohol was not degraded as quickly as with WMB. AUCs of serum ethanol levels were much greater in the control group than EMB and SKA, as much as the positive-control in both trials and the AUSs were much smaller in WMB than the positive-control. AUC of serum acetaldehyde levels were lower 
Table I. Contents of phytochemicals in mulberry extracts using water and ethanol.

\begin{tabular}{lcc}
\hline Component & $\begin{array}{c}\text { Water extract } \\
(\mathrm{n}=3)\end{array}$ & $\begin{array}{c}\text { Ethanol extract } \\
(\mathrm{n}=3)\end{array}$ \\
\hline Quercetin & $0.18 \pm 0.08$ & $0^{\mathrm{a}}$ \\
Hydroxybenzoic acid & $3.66 \pm 0.18$ & $4.22 \pm 0.21$ \\
Genestic acid & $0.10 \pm 0.0$ & $0.23 \pm 0.05^{\mathrm{a}}$ \\
Rutin & $8.6 \pm 0.00$ & $0.25 \pm 0.04^{\mathrm{b}}$ \\
GABA & $0.0018 \pm 0.0$ & $0.008 \pm 0.0^{\mathrm{a}}$ \\
Luteolin & $0.01 \pm 0.0$ & 0 \\
Cinnamic acid & $0.41 \pm 0.06$ & $0^{\mathrm{a}}$ \\
Cyanidin-3-glucoside & $6.45 \pm 0.03$ & $5.94 \pm 0.09^{\mathrm{a}}$ \\
\hline
\end{tabular}

Values represented mean \pm standard deviation. ${ }^{\mathrm{a}} \mathrm{P}<0.05$ vs. water extract group; ${ }^{\mathrm{b}} \mathrm{P}<0.01 \mathrm{vs}$. water extract group.

Table II. Contents of amino acids in silk protein hydrolysate.

\begin{tabular}{lc}
\hline Amino acids & Contents $(\%)$ \\
\hline Aspartate & 0.48 \\
Serine & 2.75 \\
Glutamate & 0.44 \\
Glycine & 8.40 \\
Histamine & 0.05 \\
Cysteine & 0.25 \\
Threonine & 0.05 \\
Valine & 0.71 \\
Methionine & 0.03 \\
Lysine & 0.07 \\
Arginine & 0.06 \\
Tyrosine & 0.28 \\
Alanine & 7.09 \\
Proline & 0.23 \\
Isoleucine & 0.18 \\
Leucine & 0.19 \\
Phenylalanine & 0.02 \\
Tryptophan & 0 \\
\hline
\end{tabular}

in the descending order of control, EMB, positive-control, SKA, and WMB and serum acetaldehyde was decreased the most in WMB (Figs. 1C, 2C). Thus, WMB and SKA reduced serum ethanol concentration as much as the positive-control and WMB decreased serum acetaldehyde more than the positive-control.

Effects of extracts on movement and short-memory after administering alcohol. At $2 \mathrm{~h}$ after alcohol administration, all rats exhibited the maximum decrease in mean movement velocity, but WMB increased the mean velocity as much as the positive-control. SKA also improved the mean velocity. At $5 \mathrm{~h}$, frequencies of the right turns were lower in the control group than the positive-control during Y maze test and WMB
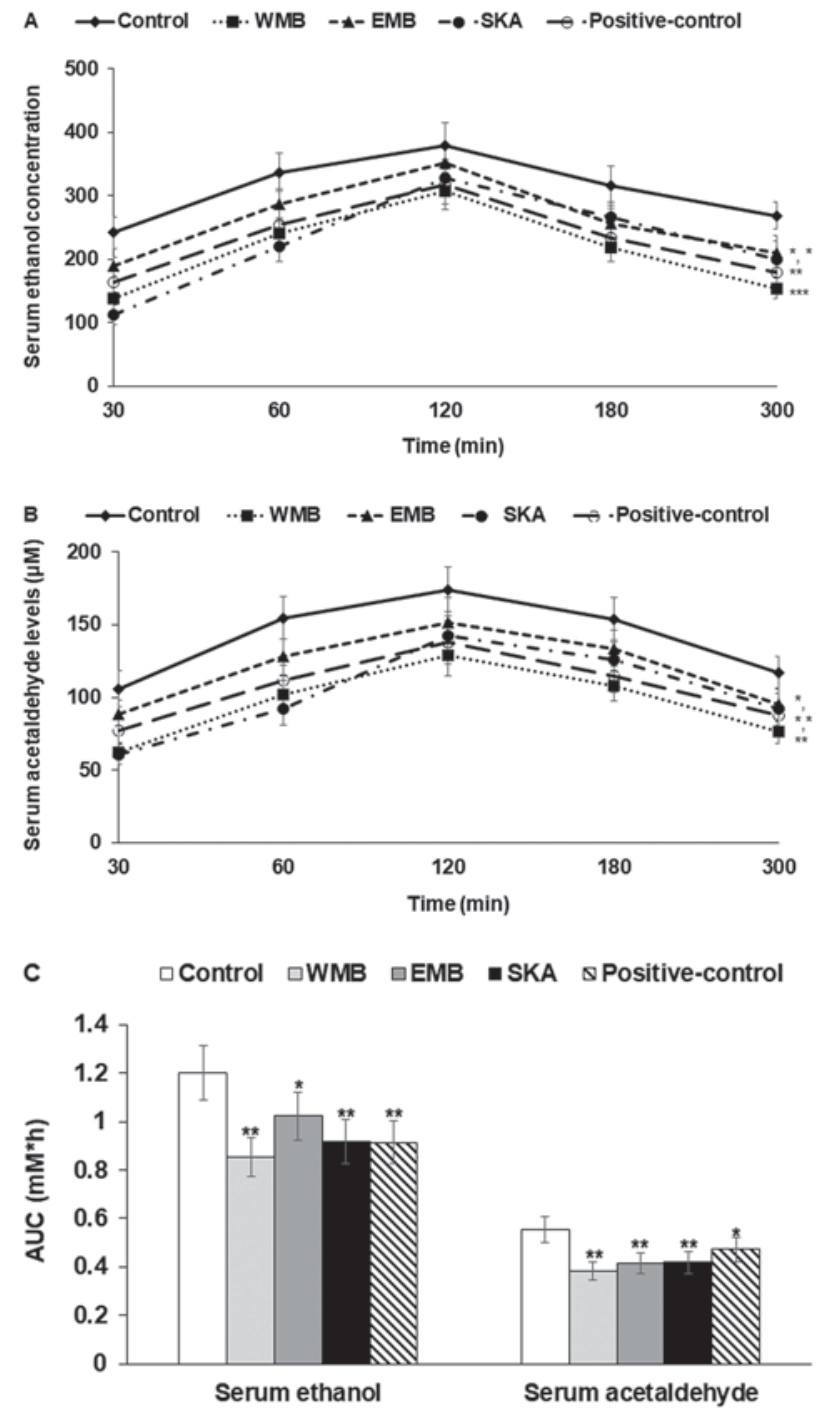

Figure 1. Serum ethanol and acetaldehyde concentrations after ethanol administration with mulberry extracts and SKA: 1st trial. (A) Changes in serum ethanol concentrations; (B) Changes in acetaldehyde concentrations; (C) Area under the curve of serum ethanol and acetaldehyde concentrations. Control, administered $0.3 \mathrm{~g}$ dextrin in $1 \mathrm{ml}$ water $(\mathrm{n}=10)$; WMB, $0.3 \mathrm{~g}$ water extract of mulberry in $1 \mathrm{ml}$ water $(\mathrm{n}=10)$, EMB, $0.3 \mathrm{~g}$ ethanol extract of mulberry $(\mathrm{n}=10)$; SKA, $0.3 \mathrm{~g}$ silk protein hydrolysates $(\mathrm{n}=10) .{ }^{*} \mathrm{P}<0.05,{ }^{* *} \mathrm{P}<0.01$, ${ }^{* * *} \mathrm{P}<0.001$ vs. control group.

increased the frequencies of the right turn to more than the positive-control (Fig. 3). SKA increased the frequencies to as much as the positive-control during the Y maze test (Fig. 3). These results indicated that alcohol administration decreased the early-time movement and short-term memory and WMB and SKA suppressed the decrease in movement and short-term memory.

Liver toxicity. Alcohol challenge increased serum levels of ALT and AST, indicators of liver toxicity, in the rats having the two alcohol challenges (Table III). WMB, EMB and SKA lowered serum AST and ALT to less than the control group. Moreover, SKA decreased the levels as much as the positive-control and WMB reduced the levels more than the positive-control (Table III). The activity of $\gamma$-GPT was also increased in the control group more than in the positive-control group, but WMB decreased $\gamma$-GPT activity more than the 

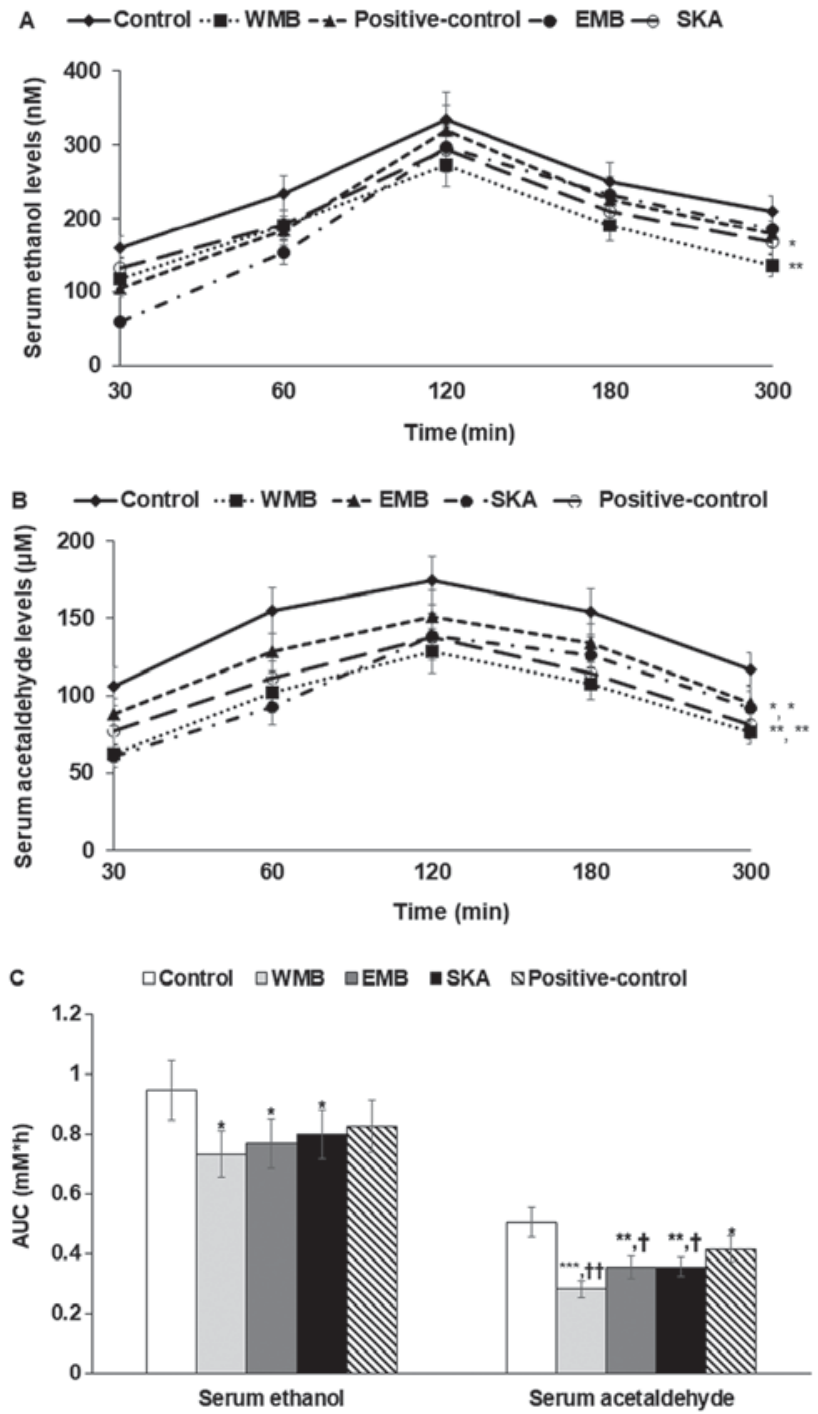

Figure 2. Serum ethanol and acetaldehyde concentrations after ethanol administration with mulberry extracts or silk protein hydrolysates: 2 nd trial. (A) Changes in serum ethanol concentrations; (B) Changes in acetaldehyde concentrations; (C) Area under the curve of serum ethanol and acetaldehyde concentrations Control, administered $0.3 \mathrm{~g}$ dextrin in $1 \mathrm{ml}$ water $(\mathrm{n}=10)$; WMB, $0.3 \mathrm{~g}$ water extract of mulberry in $1 \mathrm{ml}$ water $(\mathrm{n}=10), \mathrm{EMB}, 0.3 \mathrm{~g}$ ethanol extract of mulberry $(\mathrm{n}=10)$; SKA, $0.3 \mathrm{~g}$ silk protein hydrolysates $(\mathrm{n}=10) .{ }^{*} \mathrm{P}<0.05,{ }^{* *} \mathrm{P}<0.01,{ }^{* * *} \mathrm{P}<0.001$ vs. control group. ${ }^{\dagger} \mathrm{P}<0.05,{ }^{\dagger} \mathrm{P}<0.01$ vs. positive-control group.

positive-control. The liver toxicity is associated with the increased levels of alcohol and acetaldehyde and oxidative stress levels in the blood circulation and intracellular cells.

Serum and cellular levels of ethanol and acetaldehyde is reported to be regulated by the gene expression levels of $\mathrm{ADH}, \mathrm{ALDH}$ and CYP2E1. WMB and EMB increased gene expression of ADH and ALDH and WMB increased it the most (Fig. 4). SKA also increased the expression of ADH but it did not elevate ALDH very much in comparison to the control (Fig. 4). Thus, SKA itself may not improve acetaldehyde induced cytotoxicity and hangover symptoms. The expression of CYP2E1, a member of the cytochrome P450 mixed-function oxidase system, which is involved in xenobiotics metabolism, was elevated by WMB, EMB and SKA, compared to the control and WMB increased its expression the most (Fig. 4). Thus, WMB and SKA may reduce liver toxicity by reducing blood and cellular ethanol and acetaldehyde concentrations.

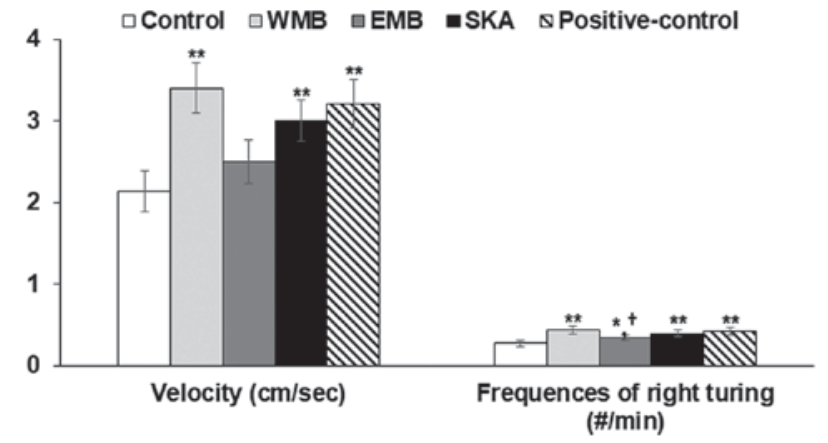

Figure 3. Voluntary movement and short-memory after ethanol administration. Voluntary movement and short-memory were measured by locomotive activity and Y-maze, respectively. Control, administered $0.3 \mathrm{~g}$ dextrin in $1 \mathrm{ml}$ water $(\mathrm{n}=10)$; WMB, $0.3 \mathrm{~g}$ water extract of mulberry in $1 \mathrm{ml}$ water $(n=10), E M B, 0.3 \mathrm{~g}$ ethanol extract of mulberry $(n=10)$; SKA, $0.3 \mathrm{~g}$ silk protein hydrolysates $(\mathrm{n}=10) .{ }^{*} \mathrm{P}<0.05,{ }^{* * *} \mathrm{P}<0.01$ vs. control group. ${ }^{\dagger} \mathrm{P}<0.05$ vs. positive-control group.

Accumulation of lipid peroxides and TG. TBARS values, representing amounts of lipid peroxides, were lower the liver and brain of WMB administered rats in comparison to the control. WMB, EMB, SKA decreased TBARS values induced by alcohol in comparison to the control and $\mathrm{WMB}(50 \mu \mathrm{g} / \mathrm{ml})$ and SKA decreased the TBARS values the most in the liver and brain (Fig. 5A). The treatments of WMB extracts and SKA also lowered the contents of lipid peroxides induced by acetaldehydes and WMB and EMB decreased them the most in the liver (Fig. 5A).

Alcohol and acetaldehyde increased TG deposition in the liver and brain cells. WMB decreased TG accumulation the most following both ethanol and acetaldehyde exposure and EMB and SKA decreased it but the decrease was not as much as WMB (Fig. 5B).

Activities of anti-oxidant enzymes and expression of pro-inflammatory cytokines in the liver. The activities of anti-oxidant enzymes, such as SOD and GSH-Px, were also associated with prevention of cytotoxicity by alcohol and acetaldehyde. All treatments increased the activities of $\mathrm{Cu} / \mathrm{Zn}$ SOD and GSH-Px in comparison to the control (Table III). The increase of their activities were similar among the treatment groups (Table III). The mRNA expression of TNF- $\alpha$ and IL-6 was lower in the descending order of the control, SKA, positive-control, EMB and WMB and WMB decreased their expressions the most (Fig. 5C).

\section{Discussion}

Alcohol consumption increases serum alcohol and acetaldehyde concentrations, which induces oxidative stress and release of proinflammatory cytokines thereby damaging some tissues, especially liver and brain $(6,22)$. Most studies have examined therapeutic strategies for alleviating alcohol-induced hepatic steatosis and gastritis caused by long-term alcohol consumption $(22,23)$. However, liver damage from short-term alcohol consumption should also be reduced to prevent alcohol-induced hepatic steatosis. People have different capacities for alcohol and acetaldehyde degradation and different rates of natural recovery from the damage due to various factors such as 
Table III. Enzyme activities related to liver toxicity and anti-oxidative system.

\begin{tabular}{|c|c|c|c|c|c|}
\hline Enzyme & Control & WMB & EMB & SKA & Positive-control \\
\hline Serum AST (U/l) & $59.4 \pm 3.9^{\mathrm{a}}$ & $41.2 \pm 3.3^{c}$ & $49.5 \pm 3.2^{\mathrm{b}}$ & $44.8 \pm 3.6^{c}$ & $43.4 \pm 3.1^{\mathrm{c}}$ \\
\hline Serum ALT (U/l) & $34.3 \pm 2.7^{\mathrm{a}}$ & $22.3 \pm 2.1^{\mathrm{c}}$ & $26.7 \pm 2.9^{\mathrm{b}}$ & $24.5 \pm 2.6^{\mathrm{b}, \mathrm{c}}$ & $25.8 \pm 2.9^{\mathrm{b}}$ \\
\hline Serum $\gamma$-GPT $(\mathrm{U} / \mathrm{l})$ & $65.4 \pm 5.8^{\mathrm{a}}$ & $52.4 \pm 4.8^{\mathrm{c}}$ & $59.5 \pm 4.4^{\mathrm{b}}$ & $57.5 \pm 4.7^{\mathrm{b}, \mathrm{c}}$ & $59.2 \pm 4.8^{\mathrm{b}}$ \\
\hline Hepatic SOD (U/mg protein) & $50.5 \pm 5.4^{\mathrm{a}}$ & $33.5 \pm 3.8^{b}$ & $35.7 \pm 4.6^{\mathrm{b}, \mathrm{c}}$ & $34.6 \pm 4.7^{c}$ & $39.5 \pm 4.5^{\mathrm{b}}$ \\
\hline Hepatic GSH-Px (U/mg protein) & $88.6 \pm 9.8^{\mathrm{a}}$ & $65.6 \pm 6.4^{c}$ & $73.5 \pm 6.6^{\mathrm{b}}$ & $66.8 \pm 6.6^{c}$ & $72.9 \pm 7.1^{\mathrm{b}}$ \\
\hline Hepatic GSH (umol/g protein) & $20.6 \pm 1.8^{c}$ & $26.4 \pm 2.3^{\mathrm{a}}$ & $23.6 \pm 2.1^{\mathrm{b}}$ & $25.6 \pm 2.3^{\mathrm{a}}$ & $24.3 \pm 2.1^{\mathrm{a}, \mathrm{b}}$ \\
\hline
\end{tabular}

Control, administered $0.3 \mathrm{~g}$ dextrin in $1 \mathrm{ml}$ water $(\mathrm{n}=10)$; WMB, $0.3 \mathrm{~g}$ water extract of mulberry in $1 \mathrm{ml}$ water $(\mathrm{n}=10)$, EMB, $0.3 \mathrm{~g}$ ethanol extract of mulberry $(\mathrm{n}=10)$; SKA, $0.3 \mathrm{~g}$ silk protein hydrolysates $(\mathrm{n}=10)$. AST, aspartate aminotransferase; ALT, alanine aminotransferase; $\gamma$-GPT, $\gamma$-glutamyl transpeptidase; SOD, superoxide dismutase; GSH, glutathione; GSH-Px, GSH peroxidase. ${ }^{\mathrm{a}-\mathrm{c}}$ Values with different superscripts were significantly different among the groups by Tukey test at $\mathrm{P}<0.05$.

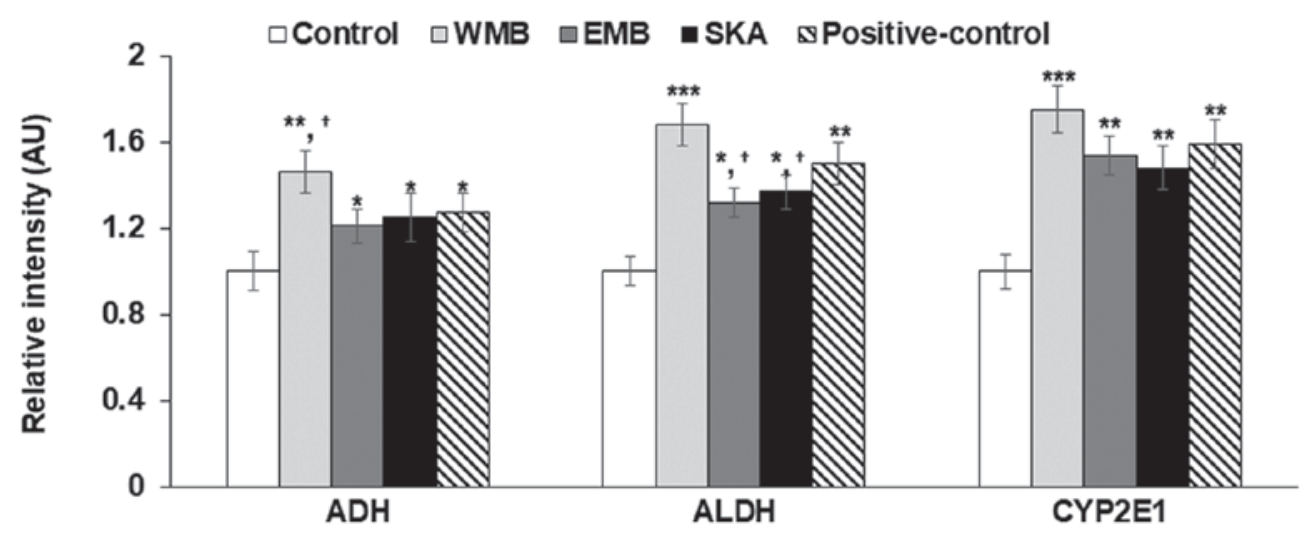

Figure 4. mRNA expression of genes involved in alcohol metabolism. Control, administered $0.3 \mathrm{~g}$ dextrin in $1 \mathrm{ml}$ water; WMB, $0.3 \mathrm{~g}$ water extract of mulberry in $1 \mathrm{ml}$ water, EMB, $0.3 \mathrm{~g}$ ethanol extract of mulberry; SKA, $0.3 \mathrm{~g}$ silk protein hydrolysates; ADH, alcohol dehydrogenase, ALDH, acetaldehyde dehydrogenase, CYP2E1, cytochrome $\mathrm{P} 450$ 2E1. ${ }^{*} \mathrm{P}<0.05,{ }^{* *} \mathrm{P}<0.01,{ }^{* * *} \mathrm{P}<0.001$ vs. control group. ${ }^{\dagger} \mathrm{P}<0.05$ vs. positive-control group.

genetics and alcohol drinking habits (24). It is important to rapidly remove alcohol and acetaldehyde from the body to reduce the damage. The degradations of alcohol and acetaldehyde are regulated by the expressions of ADH and ALDH. Alcohol consumption itself increases the expression of the enzymes. Some herbs such as fruits of Hovenia dulcis Thunb. and soybean sprouts, are known to increase their expression and they are used in products to reduce hangover (11). Since alcohol induced hangover results in not only acute problems like headache and vomiting, but also hepatic steatosis and gastritis with repetition of alcohol consumption, Therefore, better interventions for alcohol induced hangover are needed.

Mulberry, the fruit of Morus alba, is rich in anthocyanins such as cyanidin-5-glycoside and flavonoids such as rutin, qurcetin, luteolin, cyanidin-5-glycoside; mulberry leaves are the preferred food of the silk worm $(25,26)$. Flavonoids are reported to alleviate alcoholic steatosis (27) whereas antocyanins reduce non-alcoholic steatosis (28). Mulberry may improve alcoholic and non-alcoholic steatosis. Mulberry fruits contained bioactive compounds that may improve alcohol metabolism by activating both ADH and ALDH. Serum alcohol concentrations are regulated by the absorption rates and degradation of ethanol into acetaldehyde. Since acetaldehyde is also toxic, its degradation to acetate is an important factor for reducing hangover. People with genetic variants that limit the activity of ALDH get severe flushing, sweating and illness from drinking even relatively small amounts of alcohol, which are similar to symptoms of hangover (29). Clearly, acetaldehyde has serious long and short-term potential for toxic effects and induce hangovers. ADH linearly degrades ethanol below the saturated ethanol concentrations of $\mathrm{ADH}$, but it has zero-order kinetics at high ethanol concentration (30). Slow absorption reduces the alcohol overload of ADH. Meanwhile, silk protein including sericin decreases fat accumulation in the liver and blood and it also reduces serum glucose levels by suppressing glucose absorption in the intestines (31-33). Thus, it is possible that mulberry fruits and silk protein may reduce serum ethanol levels by either suppressing ethanol absorption or increasing ethanol degradation.

Alcohol is known to be readily absorbed throughout the gastrointestinal tract, but the rate of absorption is affected by several factors such as fasting and fed states, foods eaten with alcohol, and the metabolism of alcohol in the gut. Solid meals reduce alcohol absorption more than liquid meals by delaying the gastric emptying. Furthermore, sucrose in alcoholic beverages drinks lower peak and area under the curve of serum ethanol concentrations after consumption of alcohol (34). This is shown to be related to delayed gastric emptying (34). In the present study, all rats were fasted for $16 \mathrm{~h}$ and WMB, EMB, SKA and dextrin were orally provided as solutions. Thus, 

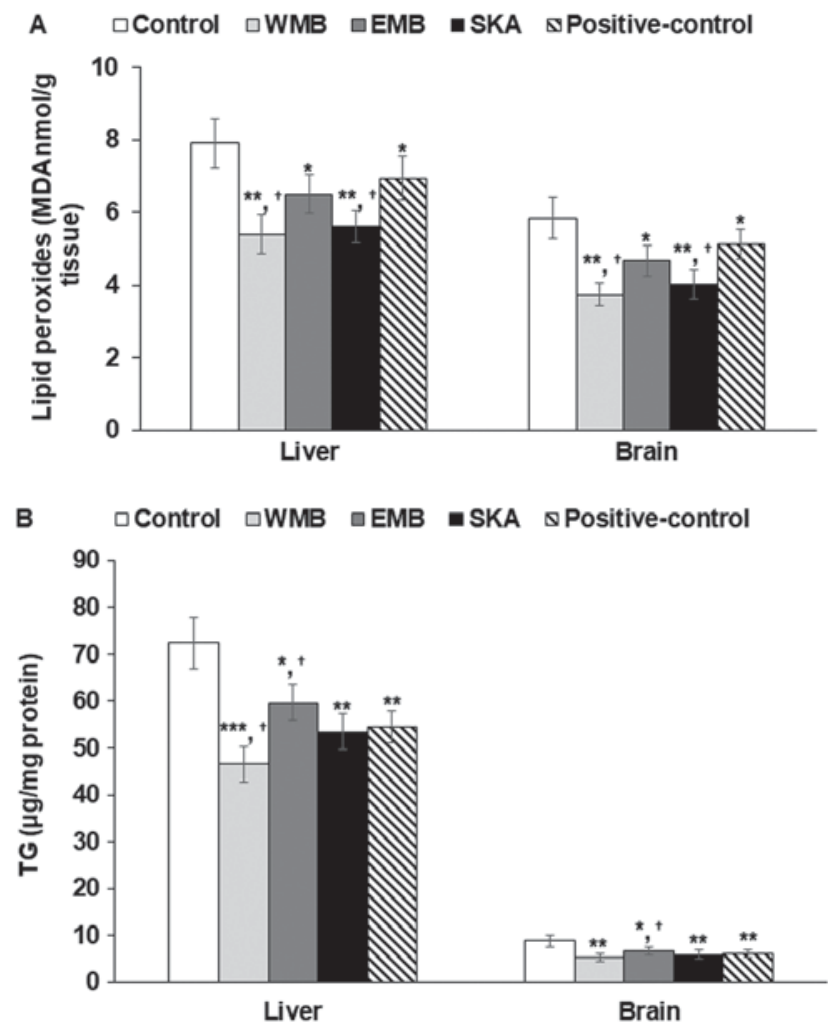

C $\square$ Control $\square$ WMB $\square$ EMB -SKA $\triangle$ Positive-control

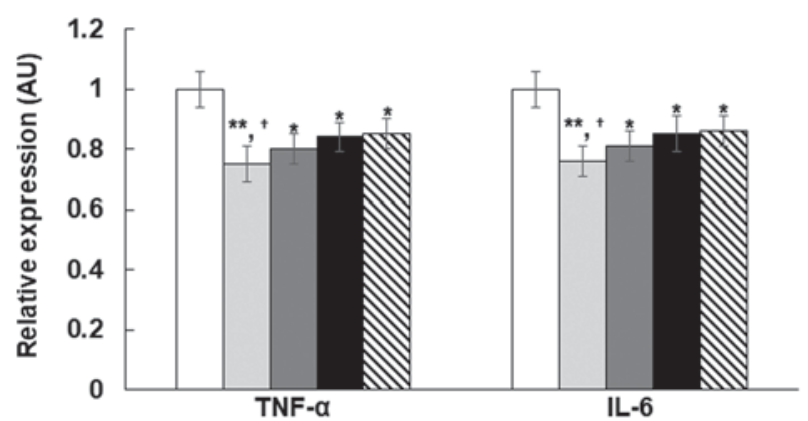

Figure 5. Lipid peroxides, triglyceride contents and mRNA expressions of genes encoding pro-inflammatory cytokines. (A) Lipid peroxides in the liver and brain; (B) Triglyceride contents in the liver and brain; (C) mRNA expressions of tumor necrosis- $\alpha$ (TNF- $\alpha$ ) and intereukin-6 (IL-6). Control, administered $0.3 \mathrm{~g}$ dextrin in $1 \mathrm{ml}$ water $(\mathrm{n}=10)$; WMB, $0.3 \mathrm{~g}$ water extract of mulberry in $1 \mathrm{ml}$ water $(\mathrm{n}=10)$, EMB, $0.3 \mathrm{~g}$ ethanol extract of mulberry $(\mathrm{n}=10)$ SKA, $0.3 \mathrm{~g}$ silk protein hydrolysates $(\mathrm{n}=10)$. ${ }^{*} \mathrm{P}<0.05,{ }^{* *} \mathrm{P}<0.01,{ }^{* * *} \mathrm{P}<0.001$ vs. control group. ${ }^{\mathrm{T}} \mathrm{P}<0.05$ vs. positive-control group.

the differences in ethanol absorption are associated with the extracts provided, and not by other foods. SKA administered rats exhibited slowly increased serum alcohol levels until $60 \mathrm{~min}$ but markedly elevated serum levels until $120 \mathrm{~min}$. This indicated that SKA delayed the absorption of ethanol more than mulberry extracts but all extract delayed absorption compared to control. The delay of gastric emptying is important, not only to reduce peak serum ethanol levels but also area under the curve of serum ethanol levels since ADH linearly degrades ethanol concentration-dependently at low levels. However, WMB quickly increased serum alcohol levels until 60 min but slowly increased the levels from $60 \mathrm{~min}$. This indicated that SKA, but not WMB, also delayed alcohol absorption to decrease serum alcohol levels. The better ethanol metabolism by WMB might be associated with higher contents of flavonoids such as rutins, luteolin, and quercetin than that of EMB. No studies about ethanol metabolism have been undertaken SKA EMB, and WMB.

After alcohol drinking most ethanol goes to the liver and is degraded into acetaldehyde. Which is eventually broken down to acetate. Ethanol and acetaldehyde intoxicate the organs, mainly liver and brain (35). Increment of ADH expression is a major way to reduce serum ethanol levels $(3,34)$. Previous studies have demonstrated that the expression levels of ADH and ALDH play a crucial role in determining the rates of lowering serum ethanol and acetaldehyde concentrations $(3,36)$. The expressions of ADH and ALDH were increased from 0.5 to $3 \mathrm{~h}$ in taraxerone treated rats in a dose and time-dependent manner after alcohol challenge, which lowered serum ethanol and acetaldehyde levels remarkably more than in DMSO treated rats (3). The present study also showed that WMB increased both $\mathrm{ADH}$ and ALDH expressions the most, and that the enzymatic activities corresponded to the rate of decrease of serum alcohol and acetaldehyde concentrations at $5 \mathrm{~h}$ after alcohol load. EMB and SKA also increased their expression more than the control but not as much as WMB.

The alcohol hangover symptoms such as headache, nausea, and dizziness are difficult to check in animal studies. However, some behaviour changes such as decreased movement and less right turns in the Y maze represent measurable alcohol hangover symptoms in rats (37). In addition, the decrease of serum ethanol and acetaldehyde levels is associated with alcohol hangover in human studies (9). The present study demonstrated that WMB and SKA decreased serum ethanol levels and increased movement and number of right turns in the $\mathrm{Y}$ maze.

Acute ethanol exposure is reported to damage cells by increasing oxidative stress due to higher lipid peroxide production and decreased hepatic GSH contents $(22,38)$. The reactive oxygen species produced by ethanol can be suppressed by rapid metabolism of ethanol and removal of reactive oxygen species. Alcohol increases reactive oxygen species production, requiring a corresponding increase the activities of antioxidant enzymes such as SOD and GSH-Px, but if the activities are not potentiated, the consequence is an increased burden of reactive oxygen species. The present study showed that the rats in the control group had liver damage, as shown by increased serum ALT and AST levels and lipid peroxides. WMB, EMB and SKA ameliorated the indexes of liver damage. WMB rats showed the least liver damage in comparison to the control. WMB and SKA both lowered serum ethanol and acetaldehyde levels and increased the expressions ADH and ALDH. In addition, WMB and SKA increased the activities of SOD and GSH-Px and GSH levels. GSH is an important antioxidant substrate for GSH-Px. Mulberry extracts and silk worm hydrolysates have also been reported to reduce oxidative stress by other investigators $(16,31,39,40)$. Cyanidin-3-glucoside has been shown to be the main ingredient of mulberry responsible for lowering serum lipid levels and hepatic lipids (40). Thus, WMB and SKA prevented liver damage partly by increasing the activities of antioxidant enzymes.

Alcohol challenge mainly induces liver toxicity but it also damages brain tissues, which may be associated with hangover. Ethanol and acetaldehyde in the blood passes through the blood-brain barrier and ethanol in the brain changes the 
mood by increasing the activity of endorphins and promoting neurotransmitter release $(41,42)$. Acetaldehyde also promotes the release of dopamine and endogenous opioid peptide, which can act on the brain opiate receptors to impact motion, perception and excitement (43). However, alcohol and acetaldehyde also cause brain damage. The present study showed that alcohol challenge increased the levels of lipid peroxide and TG deposition, indicating that brain tissue had some damage. WMB and SKA lowered the lipid peroxide contents and TG accumulation compared to the control. Thus, although alcohol promotes the release of neurotransmitters that temporarily improve metabolism, it also induces brain damage.

This study has some limitation due to the use of an animal model which cannot fully mimic alcoholic toxicity in humans, but allows for some determinations not possible in humans. Acute alcoholic toxicity, is characterized by numerous symptoms and pathologies, including: Fatigue, head and muscle aches, nausea, dizziness, poor concentration, depression, anxiety and more. Factors that contribute to the symptoms include dehydration, electrolyte imbalance, gastrointestinal disturbances and inflammation (44). It was not possible to assess all of the symptoms and causative pathologies in this study. However, it is clear that decreasing blood concentrations of alcohol and acetaldehyde helps alleviate the toxicity and many of the alcohol-induced symptoms and causative factors could be objectively evaluated that lead to alcohol toxicity. Finally, within-subject comparisons cannot be conducted since two alcohol challenge trials were done in the animals in the same assigned groups. Further study is needed to find the efficacy of WMB and SKA in within-subject comparison.

In conclusion, alcohol challenge increased lipid peroxides in the liver and brain and elevated serum AST and ALT levels. WMB and SKA decreased serum ethanol and acetaldehyde concentrations in comparison to the control and the decrease was as much as the positive-control. WMB and SKA decreased lipid peroxides in the liver and brain tissues by increasing the mRNA expression of ADH and ALDH and promoted the activities of SOD and GSH-Px. WMB and SKA also lowered the levels of TNF- $\alpha$ and IL-6. In addition, SKA slowed the absorption of ethanol after ethanol challenge WMB increased the degradation of ethanol and acetaldehyde mainly by rutin, quercetins luteolins. These results suggested that the combination of SKA and WMB (2.5 g/day in human equivalent) may improve acute alcohol-induced liver and brain damage, which might reduce hangover symptoms in humans. Since SKA delayed the absorption of ethanol in the gastrointestinal tract and WMB accelerated ethanol metabolism, the mixture of SKA and WMB might have better efficacy to reduce serum ethanol levels and hangover after alcohol consumption than either alone. However, the best dosage of SKA and WMB combination needs to be determined and further study is required. We will conduct another study to explore the best dosage of SKA and WMB for reducing serum alcohol levels after alcohol challenge.

\section{Acknowledgements}

The authors would like to thank Worldway Co., Ltd. (Sejong, Korea) for providing the mulberry extracts and silk protein hydrolysates.

\section{Funding}

This study was supported by the Ministry of Trade, Industry and Energy (MOTIE) and Korea Institute for Advancement of Technology (KIAT) through the Research and Development for Regional Industry (grant no. R0006422).

\section{Availability of data and materials}

The datasets generated during the study are available from the corresponding author on reasonable request.

\section{Authors' contributions}

SP and HJY designed the research and wrote the draft manuscript. MJK and ESK performed the biochemical assays and analyzed the data. DSK conducted animal study. All authors read and approved the final manuscript.

\section{Ethics approval and consent to participate}

All surgical and experimental procedures were performed according to the guidelines and with the approval of the Animal Care and Use Review Committee at Hoseo University, Korea (2013-05).

\section{Consent for publication}

Not applicable.

\section{Competing interests}

The authors declare that they have no competing interests.

\section{References}

1. Chi YC, Lee SL, Lai CL, Lee YP, Lee SP, Chiang CP and Yin SJ: Ethanol oxidation and the inhibition by drugs in human liver, stomach and small intestine: Quantitative assessment with numerical organ modeling of alcohol dehydrogenase isozymes. Chem Biol Interact 258: 134-141, 2016.

2. French SW: Chronic alcohol binging injures the liver and other organs by reducing NAD ${ }^{+}$levels required for sirtuin's deacetylase activity. Exp Mol Pathol 100: 303-306, 2016.

3. Sung CK, Kim SM, Oh CJ, Yang SA, Han BH and Mo EK: Taraxerone enhances alcohol oxidation via increases of alcohol dehyderogenase (ADH) and acetaldehyde dehydrogenase (ALDH) activities and gene expressions. Food Chem Toxicol 50: 2508-2514, 2012.

4. Shi P, Chen B, Chen C, Xu J, Shen Z, Miao X and Yao H: Honey reduces blood alcohol concentration but not affects the level of serum MDA and GSH-Px activity in intoxicated male mice models. BMC Complement Altern Med 15: 225, 2015.

5. Younossi Z and Henry L: Contribution of alcoholic and nonalcoholic fatty liver disease to the burden of liver-related morbidity and mortality. Gastroenterology 150: 1778-1785, 2016.

6. Enrico P and Diana M: On the accuracy of in vivo ethanol and acetaldehyde monitoring, a key tile in the puzzle of acetaldehyde as a neuroactive agent. Front Behav Neurosci 11: 97, 2017.

7. Song BJ, Akbar M, Jo I, Hardwick JP and Abdelmegeed MA: Translational implications of the alcohol-metabolizing enzymes, including cytochrome P450-2E1, in alcoholic and nonalcoholic liver disease. Adv Pharmacol 74: 303-372, 2015.

8. Polavarapu R, Spitz DR, Sim JE, Follansbee MH, Oberley LW, Rahemtulla A and Nanji AA: Increased lipid peroxidation and impaired antioxidant enzyme function is associated with pathological liver injury in experimental alcoholic liver disease in rats fed diets high in corn oil and fish oil. Hepatology 27: 1317-1323, 1998. 
9. Lee MH, Kwak JH, Jeon G, Lee JW, Seo JH, Lee HS and Lee JH: Red ginseng relieves the effects of alcohol consumption and hangover symptoms in healthy men: A randomized crossover study. Food Funct 5: 528-534, 2014.

10. Lee HS, Isse T, Kawamoto T, Baik HW, Park JY and Yang M: Effect of Korean pear (Pyruspyrifolia cv. Shingo) juice on hangover severity following alcohol consumption. Food Chem Toxicol 58: 101-106, 2013.

11. Yim DS, Lee KH, Jang IJ, Shin S, Lee YS and Park SC: Effect of aspartate and asparagine on metabolism and central nervous system effect of alcohol in healthy male volunteers. Kor J Pharmacol 31: 261-269, 2009

12. Ko BS, Jang JS, Hong SM, Kim DW, Sung SR, Park HR, Lee JE, Jeon WK and Park S: Effect of new remedies mainly comprised of Hovenia dulcis Thunb on alcohol degradation and liver protection in Sprague Dawley male rats. J Korean Soc Food Sci Nutr 35: 828-834, 2006

13. Jiang Z, Chen C, Wang J, Xie W, Wang M, Li X and Zhang X: Purple potato (Solanum tuberosum L.) anthocyanins attenuate alcohol-induced hepatic injury by enhancing antioxidant defense. J Nat Med 70: 45-53, 2016.

14. Hou Z, Qin P and Ren G: Effect of anthocyanin-rich extract from black rice (Oryza sativa L. Japonica) on chronically alcohol-induced liver damage in rats. J Agric Food Chem 58: 3191-3196, 2010.

15. Li YG, Ji DF, Chen S and Hu GY: Protective effects of sericin protein on alcohol-mediated liver damage in mice. Alcohol Alcohol 43: 246-253, 2008

16. Raghavendra R, Neelagund S, Kuluvar G, Bhanuprakash V and Revanaiah Y: Protective effect of partially purified $35 \mathrm{kDa}$ protein from silk worm (Bombyx mori) fecal matter against carbon tetrachloride induced hepatotoxicity and in vitro anti-viral properties. Pharm Biol 48: 1426-1431, 2010.

17. Andallu B and Varadacharyulu NC: Gluconeogenic substrates and hepatic gluconeogenic enzymes in streptozotocin-diabetic rats: Effect of mulberry (Morus indica L.) leaves. J Med Food 10: 41-48, 2007.

18. Golub HM, Zhou QG, Zucker H, McMullen MR Kokiko-Cochran ON, Ro EJ, Nagy LE and Suh H: Chronic alcohol exposure is associated with decreased neurogenesis, aberrant integration of newborn neurons, and cognitive dysfunction in female mice. Alcohol Clin Exp Res 39: 1967-1977, 2015.

19. Park S, Kim DS, Kang S and Shin BK: Synergistic topical application of salt-processed Phellodendron amurense and Sanguisorba officinalis Linne alleviates atopic dermatitis symptoms by reducing levels of immunoglobulin $\mathrm{E}$ and pro-inflammatory cytokines in NC/Nga mice. Mol Med Rep 12: 7657-7664, 2015.

20. Tripathi T, Abdi M and Alizadeh $\mathrm{H}$ : Protease-activated receptor 2 (PAR2) is upregulated by Acanthamoeba plasminogen activator (aPA) and induces proinflammatory cytokine in human corneal epithelial cells. Invest Ophthalmol Vis Sci 55: 3912-3921, 2014.

21. Livak KJ and Schmittgen TD: Analysis of relative gene expression data using real-time quantitative PCR and the 2(-Delta Delta C(T)) method. Methods 25: 402-408, 2001.

22. Fernández-Checa JC: Alcohol-induced liver disease: When fat and oxidative stress meet. Ann Hepatol 2: 69-75, 2003.

23. Yang HJ, Kim MJ, Kwon DY, Kang ES, Kang S and Park S: Gastroprotective actions of Taraxacum coreanum Nakai water extracts in ethanol-induced rat models of acute and chronic gastritis. J Ethnopharmacol 208: 84-93, 2017.

24. Wall TL, Luczak SE and Hiller-Sturmhöfel S: Biology, genetics, and environment: underlying factors influencing alcohol metabolism. Alcohol Res 38: 59-68, 2016.

25. Cha JY, Jung HJ, Jeong JJ, Yang HJ, Kim YT and Lee YS: Effects of amino acids on the activities of alcohol metabolizing enzyme alcohol dehydrogenase (ADH) and acetaldehyde dehydrogenase (ALDH). J Life Sci 19: pp1321-1327, 2009.

26. Raman ST, Ganeshan AK, Chen C, Jin C, Li SH, Chen HJ and Gui Z: In vitro and in vivo antioxidant activity of flavonoid extracted from mulberry fruit (Morus alba L.). Pharmacogn Mag 12: 128-133, 2016.
27. Kim JW, Kim TB, Kim HW, Park SW, Kim HP and Sung SH Hepatoprotective flavonoids in Opuntia ficus-indica fruits by reducing oxidative stress in primary rat hepatocytes. Pharmacogn Mag 13: 472-476, 2017.

28. Valenti L, Riso P, Mazzocchi A, Porrini M, Fargion S and Agostoni C: Dietary anthocyanins as nutritional therapy for nonalcoholic fatty liver disease. Oxid Med Cell Longev 2013: 145421,2013

29. Swift R and Davidson D: Alcohol hangover: Mechanisms and mediators. Alcohol Health Res World 22: 54-60, 1998.

30. Mitchell MC Jr, Teigen EL and Ramchandani VA: Absorption and peak blood alcohol concentration after drinking beer, wine, or spirits. Alcohol Clin Exp Res 38: 1200-1204, 2014.

31. Ampawong S, Isarangkul D and Aramwit P: Sericin ameliorated dysmorphic mitochondria in high-cholesterol diet/streptozotocin rat by antioxidative property. Exp Biol Med (Maywood) 242: 411-421, 2017.

32. Lee SH, Park D, Yang G, Bae DK, Yang YH, Kim TK, Kim D, Kyung J, Yeon S, Koo KC, et al: Silk and silkworm pupa peptides suppress adipogenesis in preadipocytes and fat accumulation in rats fed a high-fat diet. Eur J Nutr 51: 1011-1019, 2012.

33. Okazaki Y, Kakehi S, Xu Y, Tsujimoto K, Sasaki M, Ogawa H and Kato N: Consumption of sericin reduces serum lipids, ameliorates glucose tolerance and elevates serum adiponectin in rats fed a high-fat diet. Biosci Biotechnol Biochem 74: 1534-1538, 2010.

34. Wu KL, Chaikomin R, Doran S, Jones KL, Horowitz M and Rayner CK: Artificially sweetened versus regular mixers increase gastric emptying and alcohol absorption. Am J Med 119: 802-804, 2006

35. Bustamante J, Karadayian AG, Lores-Arnaiz S and Cutrera RA: Alterations of motor performance and brain cortex mitochondrial function during ethanol hangover. Alcohol 46: 473-479, 2012.

36. Yoo YM, Jung EM, Kang HY, Choi IG, Choi KC and Jeung EB: The sap of Acer okamotoanum decreases serum alcohol levels after acute ethanol ingestion in rats. Int J Mol Med 28: 489-495, 2011.

37. Asorey LG, Carbone S, Gonzalez BJ and Cutrera RA: Behavioral effects of the combined use of alcohol and energy drinks on alcohol hangover in an experimental mice model. Neurosci Lett 670: 1-7, 2018

38. Chen Y, Dong H, Thompson DC, Shertzer HG, Nebert DW and Vasiliou V: Glutathione defense mechanism in liver injury: Insights from animal models. Food Chem Toxicol 60: 38-44, 2013.

39. Srikanta AH, Kumar A, Sukhdeo SV, Peddha MS and Govindaswamy $\mathrm{V}$ : The antioxidant effect of mulberry and jamun fruit wines by ameliorating oxidative stress in streptozotocin-induced diabetic Wistar rats. Food Funct 7: 4422-4431, 2016.

40. Wu T, Yin J, Zhang G, Long $\mathrm{H}$ and Zheng X: Mulberry and cherry anthocyanin consumption prevents oxidative stress and inflammation in diet-induced obese mice. Mol Nutr Food Res 60: 687-694, 2016

41. Wen DC, Hu XY, Wang YY, Luo JX, Lin W, Jia LY and Gong XY: Effects of aqueous extracts from Panax ginseng and Hippophae rhamnoides on acute alcohol intoxication: An experimental study using mouse model. J Ethnopharmacol 192: 67-73, 2016.

42. Deehan GA Jr, Hauser SR, Wilden JA, Truitt WA and Rodd ZA: Elucidating the biological basis for the reinforcing actions of alcohol in the mesolimbic dopamine system: The role of active metabolites of alcohol. Front Behav Neurosci 7: 104, 2013.

43. Deehan GA Jr, Engleman EA, Ding ZM, McBride WJ and Rodd ZA: Microinjections of acetaldehyde or salsolinol into the posterior ventral tegmental area increase dopamine release in the nucleus accumbens shell. Alcohol Clin Exp Res 37: 722-729, 2013.

44. Salaspuro M: Acetaldehyde as a common denominator and cumulative carcinogen in digestive tract cancers. Scand J Gastroenterol 44: 912-925, 2009 